\title{
Determining Vertical Water Velocities from Seaglider
}

\author{
ELEANOR FRAJKA-WILLIAMS \\ National Oceanography Centre, Southampton, United Kingdom \\ Charles C. ERiksen And Peter B. Rhines \\ School of Oceanography, University of Washington, Seattle, Washington \\ RAMSEY R. HARCOURT \\ Applied Physics Laboratory, University of Washington, Seattle, Washington
}

(Manuscript received 13 October 2010, in final form 5 April 2011)

\begin{abstract}
Vertical velocities in the world's oceans are typically small, less than $1 \mathrm{~cm} \mathrm{~s}^{-1}$, posing a significant challenge for observational techniques. Seaglider, an autonomous profiling instrument, can be used to estimate vertical water velocity in the ocean. Using a Seaglider's flight model and pressure observations, vertical water velocities are estimated along glider trajectories in the Labrador Sea before, during, and after deep convection. Results indicate that vertical velocities in the stratified ocean agree with the theoretical Wentzel-KramersBrillouin (WKB) scaling of $w$; and in the turbulent mixed layer, scale with buoyancy, and wind forcing. It is estimated that accuracy is to within $0.5 \mathrm{~cm} \mathrm{~s}^{-1}$. Because of uncertainties in the flight model, velocities are poor near the surface and deep apogees, and during extended roll maneuvers. Some of this may be improved by using a dynamic flight model permitting acceleration and by better constraining flight parameters through pilot choices during the mission.
\end{abstract}

\section{Introduction}

In most places in the world's oceans, vertical velocities are small (about $1 \mathrm{~cm} \mathrm{~s}^{-1}$ ). Vertical stratification, which is typically much higher than horizontal stratification, creates a barrier to vertical motion. Exceptions to the low vertical velocity regime include vertically mixed regions, for example, wind- or convectively driven mixed layers, which can have episodes of vertical velocity faster than $10 \mathrm{~cm} \mathrm{~s}^{-1}$. In this paper, we describe a technique for estimating vertical velocities from Seaglider, an autonomous underwater vehicle, to better than $1 \mathrm{~cm} \mathrm{~s}^{-1}$ (Frajka-Williams 2009). This technique is similar to that given by Merckelbach et al. (2010) for a Slocum glider. The flight equations for the two glider models are similar; however, the number of flight parameters that are allowed to vary differs, and several relevant engineering details are different.

Corresponding author address: Eleanor Frajka-Williams, National Oceanography Centre, Empress Dock, Southampton SO14 3ZH, United Kingdom.

E-mail: e.frajka-williams@noc.ac.uk
Seaglider estimates vertical velocities $w_{w}$ from the difference between a predicted glider flight speed in still water $\left(w_{\text {stdy }}\right)$ and the observed glider vertical velocity from pressure ( $\left.w_{\text {meas }}\right)$

$$
w_{w}=w_{\text {meas }}-w_{\text {stdy }},
$$

where $w_{\text {meas }}=d z_{g} / d t$ is measured glider vertical velocity. Glider vertical position $z_{g}$, which is measured positive upward, is computed from observed pressure, and $t$ is time. The determination of $w_{\text {stdy }}$ is the subject of this paper.

The dataset used for this paper is from the Labrador Sea, where two Seagliders (sg014 and sg015) were deployed in the fall of 2004. They observed stratified ocean as well as regions convectively mixed to $1000-\mathrm{m}$ depth.

In section 2, Seaglider specifications and the dataset are described. Vertical flight speed $w_{\text {stdy }}$ is calculated by solving a flight model for the glider, which is introduced in section 3. Several parameters of the flight model need to be determined for each Seaglider, which is the subject of section 4. In section 5, we describe the observations of vertical velocity, comparing them with theoretical 


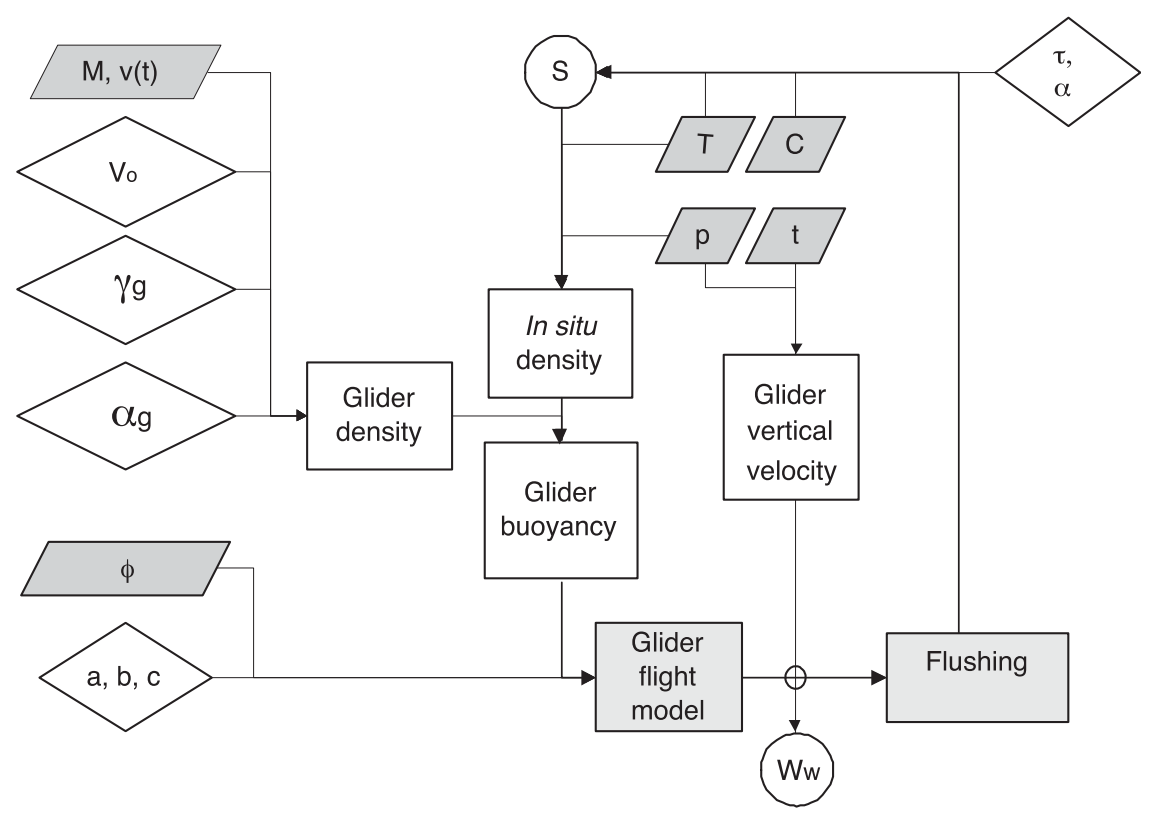

FIG. 1. Flowchart of Seaglider flight model and processing procedures. Measured quantities [shaded parallelograms; glider mass $M$, controlled volume $v(t)$, temperature $T$, conductivity $C$, pressure $p$, time $t$ and pitch $\theta$ ], parameters of the flight model (diamonds; volume $V_{0}$, glider absolute compressibility $\gamma_{g}$, thermal expansivity $\alpha_{g}$ and lift $a$, drag $b$, and induced drag $c$ coefficients), and the salinity calibration parameters $(\tau$ and $\alpha)$. The applied models, the Seaglider steady flight model, and the conductivity-temperature (CT) cell flushing speed (shaded rectangles) and calculated quantities (white rectangles) are shown. Vertical water velocity $(w)$ and salinity $(S)$ are the calculated products affected by tuning the flight model.

expectations for vertical velocity in the ocean and provide several estimates of accuracy of the technique.

\section{Seaglider}

Full details of the Seaglider engineering and flight model were introduced by Eriksen et al. (2001). Relevant information for the vertical velocity determination is repeated here.

\section{a. Glider specifications}

Seaglider is an autonomous profiling vehicle that is capable of making observations to $1000 \mathrm{~m}$ on long-duration missions. Its efficiency results from a pressure hull with nearly the same compressibility as seawater, a low-drag hydrodynamic shape, and the limited number and low power consumption of instruments. Sampling patterns are selected by the user, but a 1:3 glide angle is typical, resulting in a speed through water of about $20 \mathrm{~cm} \mathrm{~s}^{-1}$ and surfacings separated by about $6 \mathrm{~km}$ for a $1000-\mathrm{m}$ dive depth. Data are reported via Iridium satellite at the end of each dive-climb pair of profiles, which is referred to as a dive cycle. The mission's duration depends on water column stratification, dive depth, and instrument sample rate. Higher stratification requires larger changes to glider buoyancy in order for the glider to dive through it. Because stratification in the ocean is highest near the surface, frequent shallow dives consume more energy per unit time than do deeper dives. Lower sampling rates use less energy.

Seaglider moves through the water by changing its buoyancy and pitch angle, and turns by rolling. The buoyancy engine changes glider volume via a variable buoyancy device (VBD) with a range of about $800 \mathrm{~cm}^{3}$, or cc. Dive steepness is related to glider pitch, which is adjusted by moving an internal weight - the battery pack-fore and aft. Pitch angle is measured by a tilt meter (Precision Navigation TCM2-80 compass). Rolls are executed by rotating the battery pack to move the glider's center of mass left and right.

Seagliders are instrumented to measure temperature, conductivity [Sea-Bird Electronics (SBE) custom sensor, SBE 4 conductivity cell], and pressure (Paine Corporation 211-75-710-05 1500PSIA). One of the strategies to limit Seaglider power consumption is to use an unpumped conductivity-temperature (CT) cell. Uncertainties in timing between temperature and conductivity measurements can result in salinity errors. Full details of the salinity algorithm are forthcoming (C. C. Eriksen 2012, unpublished manuscript). Measured quantities used in estimating glider flight speed are shown in Fig. 1. 


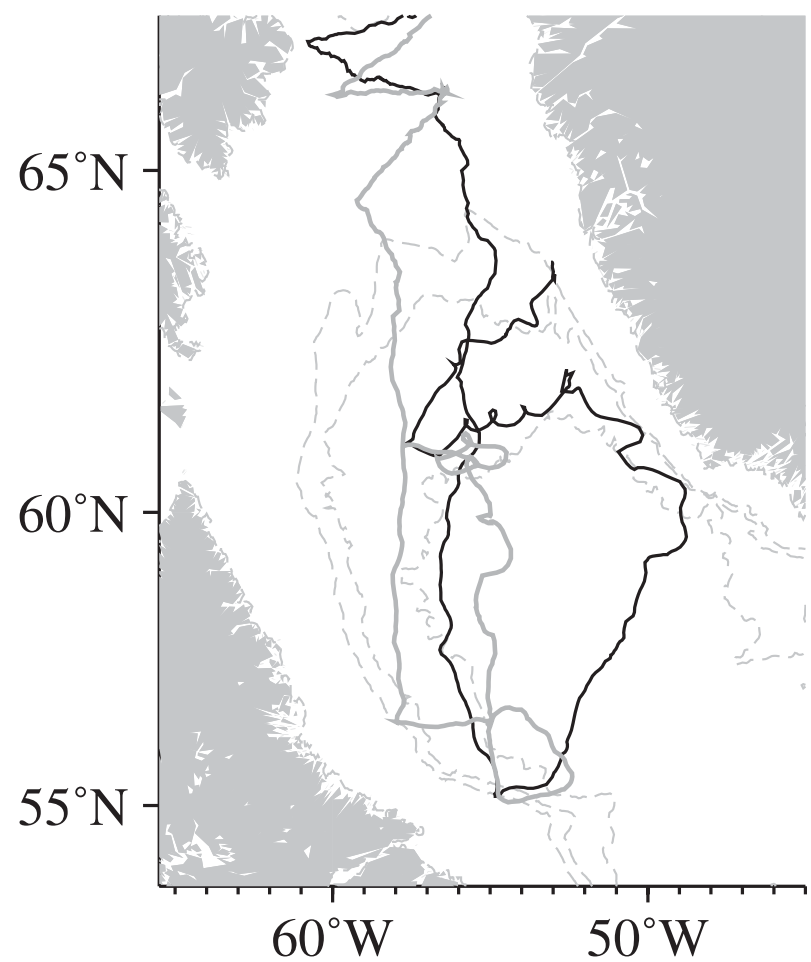

FIG. 2. Map of glider tracks: sg014 (black) and sg015 (gray). Bathymetry is contoured at a $1000-\mathrm{m}$ interval.

\section{b. Dataset}

Data used for this paper were taken from two Seagliders ( $\mathrm{sg} 014$ and sg015) that traversed the Labrador Sea between September 2004 and April 2005 (see Fig. 2). They were deployed in the Davis Strait and then transited south along meridians from $65^{\circ} \mathrm{N}$. Once they reached the $1000-\mathrm{m}$ isobath at the Labrador shelf, they crossed the region of deep convection in the central Labrador Sea (February 2005), before returning toward Nuuk, Greenland, for recovery.

Each glider sampled temperature, conductivity, and pressure. The sampling rate was highest in the shallow part of the profile and lowest near the 1000-m apogee: approximately every $5 \mathrm{~s}$ in the top $150 \mathrm{~m}$ (approximately every $0.6 \mathrm{~m}$ vertically spaced), incrementally reducing to every $40 \mathrm{~s}$ from 250 to $1000 \mathrm{~m}$ (approximately every $2.4 \mathrm{~m}$ ). Estimates of salinity, temperature, and pressure are used to determine seawater density $\rho$, from which stratification can be calculated. Buoyancy frequency $N$ is determined from stratification as $N^{2}=-g \rho^{-1} \partial \sigma_{z}(z) / \partial z$, where $\sigma_{z}(z)$ is the potential density at depth $z$ and $g$ is the gravitational acceleration. Because of spikes in $\sigma_{z}$, resulting from the unpumped CT cell, $N$ is calculated as a piecewise linear fit to $-g \rho^{-1} d \sigma_{z}(z) / d z$ over 20 -m bins.

An additional data product for surface fluxes will be used to verify vertical velocity measurements. Surface winds and buoyancy flux from National Centers for Environmental
Prediction (NCEP)/Department of Energy Global Reanalysis 2 data provided by the National Oceanic and Atmospheric Administration (NOAA)/Office of Oceanic and Atmospheric Research (OAR)/Earth System Research Laboratory (ESRL)/Physical Sciences Division (PSD), Boulder, Colorado, were downloaded from their Web site at http://www.esrl.noaa.gov/psd (Kanamitsu et al. 2002). We used the 6-hourly product at $2.5^{\circ}$ resolution.

\section{Flight model}

The Seaglider flight model assumes lift, drag, and buoyancy forces. Lift and drag parameterizations were determined from its hydrodynamic shape (Eriksen et al. 2001; Hubbard 1980). The forces are lift $L, \operatorname{drag} D$, and buoyancy $B$,

$$
\begin{aligned}
& L=q l^{2} a \alpha, \\
& D=q l^{2}\left(b q^{-1 / 4}+c \alpha^{2}\right), \\
& B=g(-M+\rho V(t, p, T),
\end{aligned}
$$

where $l$ is the hull length (1.8 $\mathrm{m}$, not including antenna), $a$ the lift coefficient, $\alpha$ the attack angle, $\theta$ the glide angle, $b$ the drag coefficient, $c$ the induced drag coefficient, $g$ the gravitational acceleration, $q$ the dynamic pressure, $M$ the glider mass, and $V(t, p, T)$ the glider volume, which depends on $t$ time, $p$ pressure, and $T$ ambient temperature. Dynamic pressure is equal to $q=\rho\left(U^{2}+W^{2}\right) / 2$ where $U$ and $W$ are horizontal and vertical glider speeds relative to water motion. Attack and glide angles are related via the pitch angle $\varphi$ as $\varphi=\alpha+\theta$.

In Eq. (4), the buoyancy force $B$ results from the difference between the mass of the glider $M$ and of the seawater displaced by the glider volume $V$. Glider volume changes in time result from the VBD, a buoyancy device that pumps oil from an internal reservoir within the glider's fixed volume to a bladder outside, effectively increasing the glider volume while maintaining a constant mass. Glider volume also depends on pressure and temperature via the glider compressibility and volumetric thermal expansion. Glider volume is given as

$$
V(t, p, T)=\left[V_{0}+v_{c}(t)\right] e^{-\gamma_{g} p+\alpha_{g}\left(T-T_{0}\right)},
$$

where $v_{c}(t)$ is the change in volume resulting from the pump; $V_{0}$ is the glider volume at $p=0$ and $T=T_{0}$, with the pump in $v_{c}(t)=0$ position; $\gamma_{g}$ and $\alpha_{g}$ are the compressibility and volumetric thermal expansion coefficients, respectively; and $T_{0}$ is an arbitrary reference temperature. In this formulation, we have approximated both the compressibility and thermal expansion as constant 
TABLE 1. Table of parameters.

\begin{tabular}{cll}
\hline \hline Parameter & \multicolumn{1}{c}{ Description } & \multicolumn{1}{c}{ Unit } \\
\hline$a$ & Lift coefficient & $\mathrm{rad}^{-1}$ \\
$b$ & Drag coefficient & $\mathrm{m}^{1 / 4} \mathrm{~kg}^{1 / 4} \mathrm{~s}^{-1 / 2}$ \\
$c$ & Induced drag coefficient & $\mathrm{rad}^{-2}$ \\
$\theta$ & Glide angle & $\mathrm{rad}$ \\
$\alpha$ & Attack angle & $\mathrm{rad}$ \\
$\varphi$ & Pitch angle & $\mathrm{rad}$ \\
$V_{0}$ & Volume at $p=0, T=T_{0}, v_{c}=0$ & $\mathrm{cc}$ \\
$v_{\mathrm{c}}$ & Change in volume resulting from & $\mathrm{cc}$ \\
& VBD & \\
$T_{0}$ & Reference temperature & ${ }^{\circ} \mathrm{C}$ \\
$\gamma_{g}$ & Glider compressibility & $\mathrm{dbar}$ \\
$\alpha_{g}$ & Glider thermal expansivity & ${ }^{\circ} \mathrm{C}-1$ \\
$M$ & Glider mass & $\mathrm{kg}^{-1}$ \\
\hline
\end{tabular}

multipliers of pressure and temperature, respectively. The choice of reference temperature $T_{0}$ is arbitrary, but a change in $T_{0}$ will affect the estimate of initial glider volume $V_{0}$. Note that $v_{c} \ll V_{0}$, so the compressibility and thermal expansion effects of the oil volume are small. Thus, buoyancy force is calculated from parameters, known constants, and in situ Seaglider measurements.

Assuming steady flight, that is, no acceleration, the forces must balance (as shown in Fig. 5)

$$
B+\cos \theta L-\sin \theta D=0 .
$$

Rearranging Eqs. (2)-(4) and (6) gives an implicit equation for $W$ :

$W=\left(\frac{\rho}{2}\right)^{3 / 4}\left(\frac{l^{2} U^{5 / 2}}{B}\right) \frac{b}{\cos ^{5 / 2} \theta}+\left(\frac{2}{\rho}\right)\left(\frac{B c}{l^{2} U}\right) \frac{\cos ^{3} \theta}{a^{2}}$.

From this it can be seen that the $W$ dependence on lift and drag coefficients may have compensating effects.

The flight Eqs. (2) and (3) are solved iteratively for $q$ and $\alpha$. From $q$ and $\alpha, U^{2}+W^{2}$ and $\theta$ are determined. Finally, the modeled vertical velocity of the glider $w_{\text {stdy }}$ is determined from $U^{2}+W^{2}$ as

$$
w_{\text {stdy }}=\sqrt{U^{2}+W^{2}} \sin \theta .
$$

Basic flight model parameters, constants, and measured quantities are summarized in Table 1. Glider data processing is diagrammed in Fig. 1, where measured quantities are in shaded parallelograms and undetermined constants and coefficients are in diamonds. The loop at the righthand side indicates a feedback between calculations of salinity and vertical velocity. However, small changes in salinity have little effect on vertical velocity.

\section{a. Flight parameters}

Undetermined constants and coefficients include the lift, drag and induced drag coefficients, volume, glider absolute compressibility, and glider volumetric thermal expansion. Nominal starting values for flight coefficients and compressibility were estimated from tank tests, pressure tests, and summing the volumes of individual glider components. Initial parameter values for sg014 were

$$
\begin{aligned}
a & =0.003836 \mathrm{rad}^{-1}, \\
b & =0.010078 \mathrm{~m}^{1 / 4} \mathrm{~kg}^{1 / 4} \mathrm{~s}^{-1 / 2}, \\
c & =2.1 \times 10^{-6} \mathrm{rad}^{-2}, \\
\gamma_{\mathrm{g}} & =4.4 \times 10^{-6} \mathrm{dbar}^{-1}, \\
V_{0} & =51400 \mathrm{cc}, \\
\alpha_{\mathrm{g}} & =70.5 \times 10^{-6}{ }^{\circ} \mathrm{C}^{-1} .
\end{aligned}
$$

Using these values, we calculated the average profiles of vertical water velocity, separated by those measured during glider dives and glider climbs (Fig. 3, dashed lines). The estimated average vertical water velocity is between 0.5 and $1 \mathrm{~cm} \mathrm{~s}^{-1}$ upward, with a $0.5 \mathrm{~cm} \mathrm{~s}^{-1}$ difference between dives and climbs, and a vertical divergence of $0.4 \mathrm{~cm} \mathrm{~s}^{-1}$ over $1000 \mathrm{~m}$. The presence of mean upwelling, and the difference between average profiles from dives as compared to climbs, indicates that glider flight parameters need to be tuned for this glider.

To demonstrate the sensitivity of vertical velocity calculations to flight parameter values, they were individually increased by $5 \%$ (or $5 \mathrm{cc}$ for volume $V_{0}$ ). The choice of $5 \%$ was arbitrary, and used to determine the sign and structure of parameter changes on $w$. For the sg015 dive cycle 230 , new profiles of vertical velocity were calculated for each increased parameter value, where the other parameters were held at the initial values. The difference profiles (new minus original) are plotted in Fig. 4. Each parameter will be discussed below.

\section{1) GLIDER VOLUMe $V_{0}$}

Volume is initially estimated as a sum of parts, but is not fully determined until the glider is in the field. A positive change to volume results in a negative change to $w_{w}$. To see how this occurs, we consider a specific example. Supposing measured glider velocity is $w_{\text {meas }}=$ $-10 \mathrm{~cm} \mathrm{~s}^{-1}$ (downward) and the flight model with initial parameters predicts a glider velocity of $w_{\text {stdy }}=-6 \mathrm{~cm} \mathrm{~s}^{-1}$. This implies a vertical water velocity of $w_{w}=w_{\text {meas }}-$ $w_{\text {stdy }}=-4 \mathrm{~cm} \mathrm{~s}^{-1}$. However, if the true glider volume is larger than the initial prediction, then the glider is more 


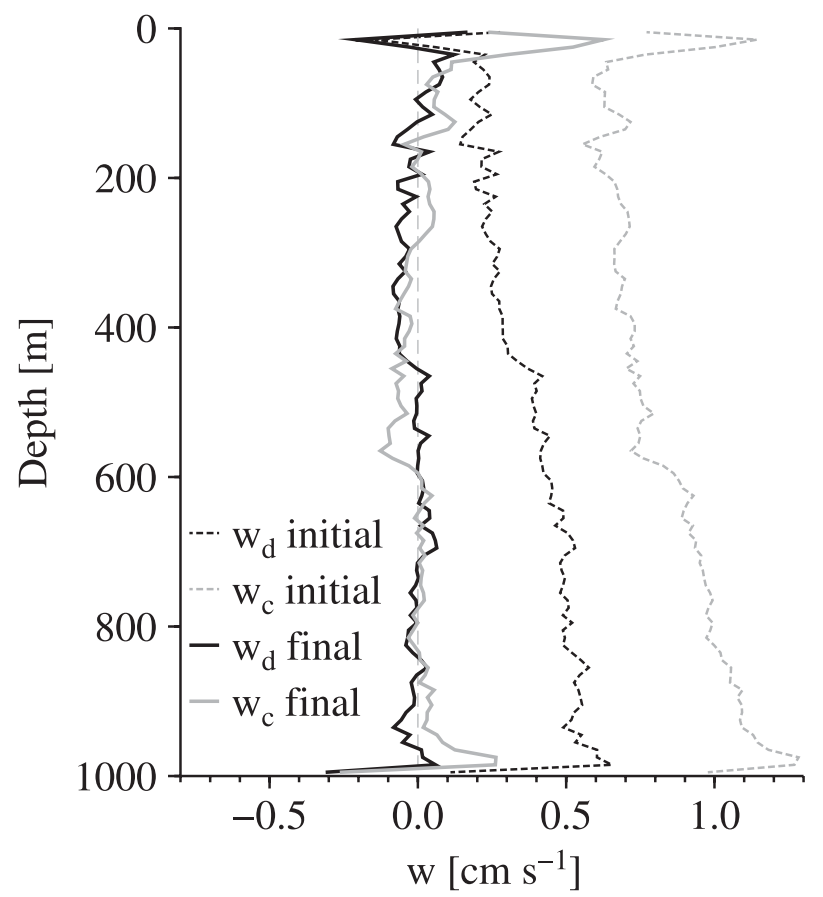

FIG. 3. Mean profiles of vertical water velocity calculated with initial flight parameters and flight parameters chosen by the minimization procedure are shown for all profiles from the sg014 mission. Mean profiles calculated from initial flight parameters (dashed lines), showing a positive mean vertical velocity, an offset between the mean profile calculated from glider dives only (black) and glider climbs only (gray), as well as a vertical convergence. Mean profiles calculated with the final set of flight parameters (solid lines). Note the near-zero mean, near-zero offset between dive and climb estimates, and the lack of vertical divergence or convergence.

buoyant everywhere than initially calculated. Adjusting glider volume by increasing $V_{0}$ to the true value results in a more upward-adjusted model-predicted glider velocity, say $w_{\text {stdy }}=-3 \mathrm{~cm} \mathrm{~s}^{-1}$. Then, the true vertical water velocity is $-7 \mathrm{~cm} \mathrm{~s}^{-1}$, which is more downward than initially expected.

The same example can be illustrated by the forcebalance diagrams of lift, drag, and glider buoyancy (Fig. 5). An increase in glider volume $V_{0}$ means that the glider is less dense and the buoyancy force $B$ in the equations will be more positive for both a dive and a climb. Then the resulting $w_{\text {stdy }}$ for both a dive and a climb will be increased, so that $w_{w}$ is decreased. We conclude that for an increase in $V_{0}$, the effect on $w_{w}$ is negative for both dives and climbs. Likewise, a decrease in $V_{0}$ results in a more positive $w_{w}$ for both dives and climbs. Furthermore, the change in $w_{w}$ is nearly depth independent.

\section{2) GLIDER COMPRESSIBILITY $\gamma_{G}$}

Seaglider compressibility was initially calculated in a pressure tank (see Fig. 4 of Eriksen et al. 2001). Below

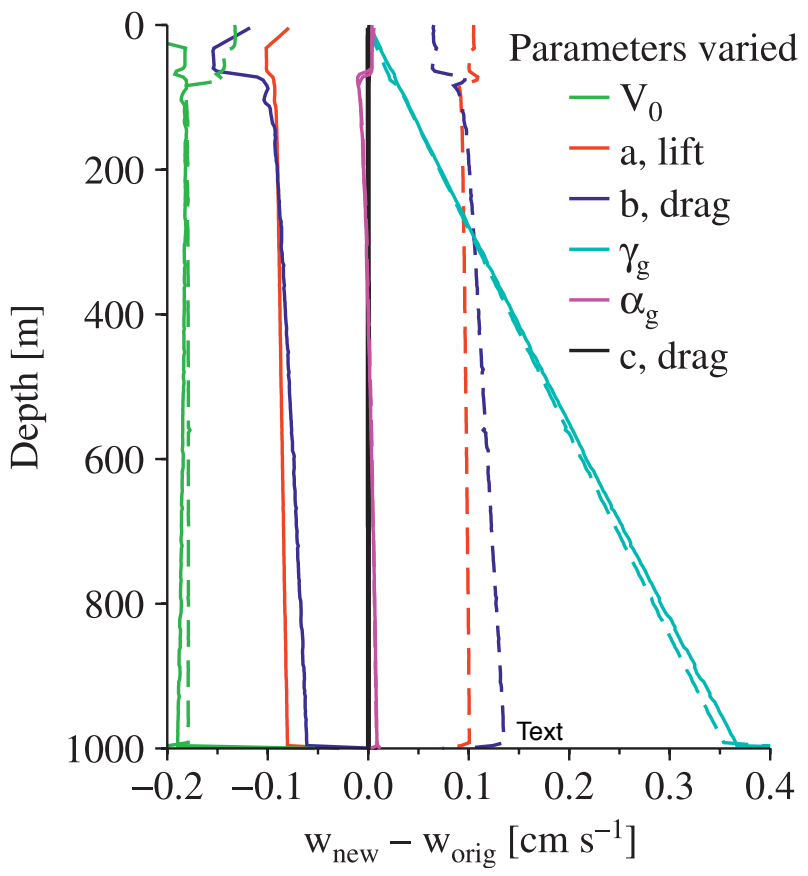

FIG. 4. The effect of increasing flight parameters on a mean profile of vertical water velocity, averaged from glider dives (solid) or climbs (dashed). The difference is shown as $w_{\text {new }}-w_{\text {orig, }}$, where $w_{\text {orig }}$ is the mean profile using a nominal parameter value and $w_{\text {new }}$ using the parameter value $\times 1.05$ (or in the case of volume, volume $+5 \mathrm{cc}$ ). The parameter being tested is indicated by color. The effect for $w_{w}$ from glider dives (solid lines) and climbs (dashed lines) are shown.

the top 20 dbar, Seaglider weight changed by less than $0.5 \mathrm{~g}$ over a 500 -dbar change in pressure for freshwater, indicating that the hull is nearly neutrally compressible. Even so, the compressibility coefficient for an individual glider depends not only on the pressure hull but on all components, including the fiberglass fairing, instruments, wings, etc.

Glider compressibility is multiplied by pressure in (5), so a change to compressibility $\gamma_{g}$ has a larger impact on vertical velocity estimates at greater depth. The result is

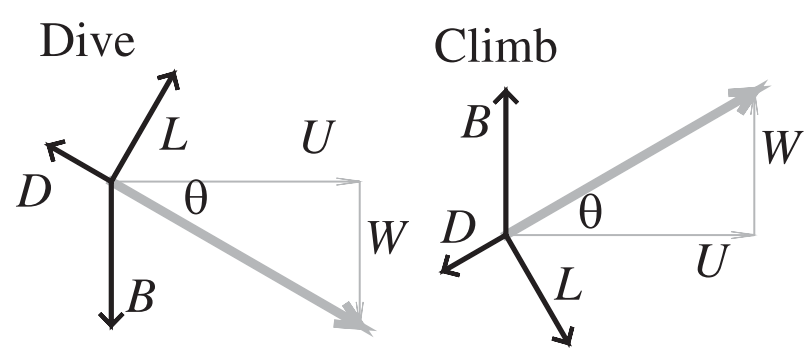

FIG. 5. Diagram of force balance on the Seaglider (left) during a dive and (right) during a climb. Forces are lift $(L)$, drag $(D)$, and buoyancy $(B)$. The glider velocity is $U$ and $W$ in the $x$ and $z$ directions, and $\theta$ is the glide angle. 
a change in vertical divergence, with the same sign effect for a dive as for a climb. An increase in compressibility means that a glider with higher compressibility is smaller (lower volume) at greater depths. An increase in compression results in lower buoyancy. Because $w_{w}=w_{\text {meas }}-$ $w_{\text {stdy }}$, if $w_{\text {stdy }}$ is more downward, then water velocity $\left(w_{w}\right)$ is more upward, as shown in Fig. 4.

\section{3) THERMAL EXPANSION $\alpha_{G}$}

In Eq. (5), glider volumetric thermal expansion $\alpha_{g}$ is multiplied both by $T$ and by $T_{0}$, an arbitrary reference temperature. Varying $\alpha_{g}$ gives rise to changes in the vertical structure of $V(t, p, T)$ through the vertical structure in $T$, but also contributes a volume offset through $\delta \alpha_{g} T_{0}$, where $\delta \alpha_{g}$ is the small change in $\alpha_{g}$. In the minimization, the effect of $\delta \alpha_{g} T_{0}$ is compensated by a change in volume $\delta V_{0}$. Once changes in $w$ resulting from $\delta \alpha T_{0}$ are compensated in this way, volumetric thermal expansion has little effect on glider flight for a $5 \%$ change in $\alpha_{g}$. For this parameter, a $5 \%$ offset is larger than the expected uncertainty in how well it is known.

\section{4) LIFT AND DRAG COEFFICIENTS: $A, B$, AND $C$}

Unlike changes in volume, the effect on $w_{w}$ resulting from a change to lift or drag coefficients differs in sign from a glider dive to a glider climb (Fig. 4). For a glider dive or climb, if the force $B \cos \theta$ is constant and $a$ increases, then the magnitude of glider speed $U^{2}+W^{2}$ must decrease. For a dive, where $w_{\text {stdy }}<0$, a decrease in the magnitude of $w_{\text {stdy }}$ means that either the glider is going down more slowly or $w_{\text {stdy }}$ is increasing (is less negative). For a climb, where $w_{\text {stdy }}>0$, a decrease in the magnitude of $w_{\text {stdy }}$ means that either the glider is going up more slowly or $w_{\text {stdy }}$ is decreasing (is less positive). For $w_{w}=$ $w_{\text {meas }}-w_{\text {stdy }}$, these changes to $w_{\text {stdy }}$ result in a decrease in calculated $w_{w}$ for a dive, and an increase in $w_{w}$ for a climb. Thus, while a nonzero mean of the profile of calculated $w_{w}$ can be adjusted by changing the volume $V_{0}$, a mean offset between dive and climb estimates of $w_{w}$ can only be adjusted by changing the lift or drag coefficients.

From these calculations and initial flight tuning efforts, it was determined that $c$ had little effect on $w$. Then, for $c \alpha^{2} \ll b q^{-1 / 4}$, we can approximate the force balance of Eqs. (2) and (3) with

$$
q l^{2}\left(b^{2} q^{-1 / 2}+\alpha^{2} a^{2}\right)^{1 / 2}=B,
$$

which shows that $b^{2}$ and $a^{2}$ can have a compensating effect. However, because $b$ and $a$ are multiplied by different variables, compensation is not exact always. While $q$ is relatively constant for a glider flight of about $20 \mathrm{~cm} \mathrm{~s}^{-1}$ through water, $\alpha$ may vary. In piloting a glider, $a$ may be varied by varying glider pitch $\varphi$, allowing $a$ and $b$ to be determined.

\section{b. Violations of the steady flight assumption}

The steady flight assumption (i.e., forces are in balance) is an approximation to true glider behavior, where acceleration occurs. To test the validity of this approximation, an unsteady version of the flight model was solved as a differential equation with time derivatives and by approximating inertia of the instrument by applying a steady flight model as a first-order process with a time constant $\tau$. The offset between $w_{w}$ from these two models was minimized for a time constant $\tau=12 \mathrm{~s}$, suggesting that over time periods longer than $12 \mathrm{~s}$ the glider is in steady state. Because the unsteady model is computationally intensive to solve in regressions, we use the steady approximation here. Periods when the steady model is not appropriate, described below, are discarded from the dataset prior to determining flight coefficients.

Differences between the steady and unsteady models are largest near the surface and deep turnaround points (apogees), where glider velocity passes through zero. Near the apogees, buoyancy and pitch change rapidly, resulting from the VBD pumping and the internal movement of the battery pack, which effects pitch changes. Air bubbles trapped in small crevices or between the pressure hull and fairing may also change the glider's compressibility near the surface. (High pressures dissolve the air.) Before determining flight parameters, we discard the $50 \mathrm{~m}$ of data around each apogee.

Glider rolling is not accounted for in the flight model. Roll maneuvers are executed by Seaglider when its measured heading differs from that intended by a userdefined threshold. The magnitude of a roll was the same for all rolls, but the duration of a roll extends until the glider measures a heading within acceptable limits. Typical roll maneuvers last a few tens of seconds. In some cases, a longer duration maneuver was required. Extended roll maneuvers appear to correlate with anomalous vertical velocity values. In Fig. 6, measured glider velocity is shown, highlighted during roll maneuvers by gray horizontal lines. The dive is on the left and the climb is on the right. Peaks in $w_{\text {meas }}$ align with the roll maneuver duration and also persist after the roll maneuver has been completed (not visible in the figure). The coincidence of vertical velocity spikes with roll maneuvers indicates an effect of rolling on glider flight that is not captured in the dynamics of the flight model. The persistence of the effect after the roll has been completed means that we cannot simply discard the period of time during roll maneuvers, but must discard the entire $w_{w}$ profile for dives with total roll durations exceeding $800 \mathrm{~s}$, before tuning the flight 


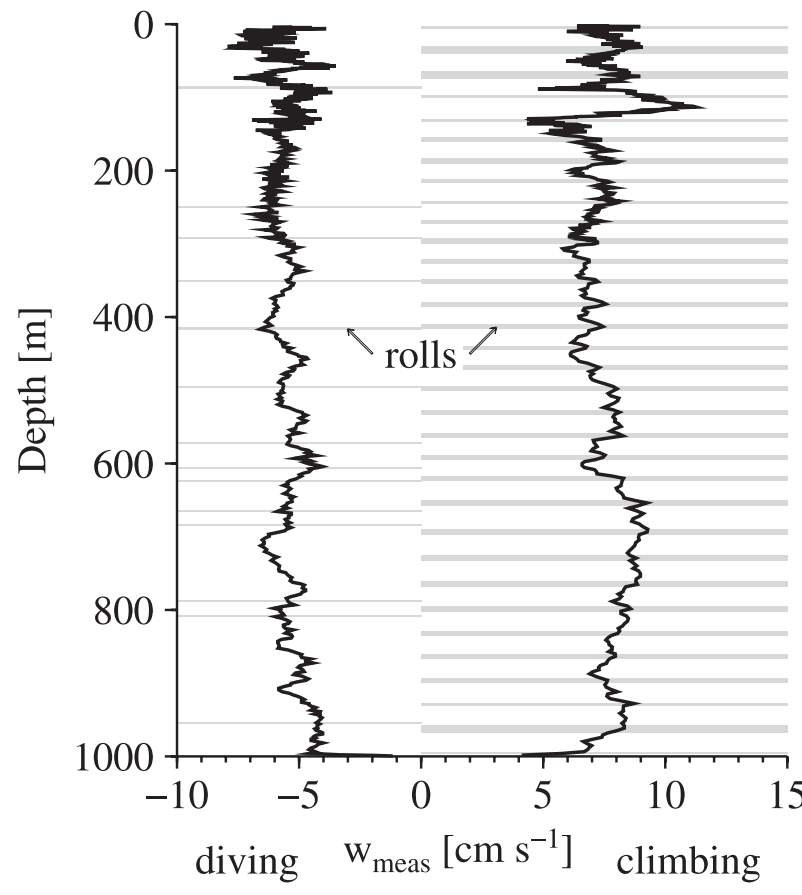

FIG. 6. Measured vertical glider velocity in the presence of roll maneuvers for sg014 dive cycle 540. Measured velocity of the Seaglider ( $\left.w_{\text {meas }}\right)$ is plotted (black); negative values indicate that the Seaglider is diving, and positive values is climbing. Periods where the glider was also rolling are shaded (gray). On the left, roll maneuvers were brief, typical of most maneuvers, but on the right they are longer duration as can be seen by the width of the gray shading.

model. The number of dives affected thus, for these two gliders, was 22 for sg015 and 8 for sg014, so the expected effect on flight parameters should be small. However, depending on how well tuned the glider roll center was, before the mission began, the incidence of extended roll maneuvers could be higher for another glider. Discarded profiles were returned to the dataset before calculating final results figures.

\section{Procedure}

To improve vertical velocity estimates, we determine the unknown flight parameters as follows: in section 4a, a cost function is chosen based on the effects of flight parameters described above, and assumptions about vertical water velocity. The cost function is then minimized over an ensemble of dive cycles, as described below in section $4 \mathrm{~b}$

\section{a. Choice of cost function for minimization}

To choose a cost function, we consider our expectations about $w_{w}$. Three primary assumptions are as follows: 1) net vertical mass transport is zero (i.e., what goes up must come down); 2) there is no mean vertical divergence; and 3) the sampling characteristics of the glider are not reflected in the measurement of $w$. Assumption 1 is mass conservation, and assumed valid over the entire ocean, but may also be appropriate on smaller space and time scales. It requires $\int w_{w}(t) d t \rightarrow 0$, where $w_{w}(t)$ is estimated water velocity at time $t$, regardless of depth. In the internal wave regime, waves are periodic in space and time. Although an individual profile of vertical velocity may have a nonzero mean depending on the phases of the waves observed, over an ensemble of randomly sampled waves, the mean should approach zero. For example, if a single $1000-\mathrm{m}$ profile sampling in an internal wave regime observes three full wavelengths and one half-wavelength of an internal wave profile, the halfwavelength will result in a nonzero mean vertical velocity over that $1000 \mathrm{~m}$. Similarly, if a climb profile samples three full wavelengths and one half-wavelength of the same sign, it will appear as though, in the average, there is net upwelling or downwelling, violating our assumption 1 . To randomize the phase of the sampled waves, it is necessary to average over a large number of dive cycles. In the mixed layer, eddying motions are primarily recirculating. Even in deep convection, convection tends to be nonpenetrative, and instead consists of vertically mixing plumes rather than net downwelling.

Assumption 2 requires no vertical divergence, which can be represented as $\int\left|w_{w}(z)-\left\langle w_{w}(z)\right\rangle_{z}\right| d z$, where $w_{w}(z)$ is a mean profile of vertical velocity averaged over an ensemble of dives, and $\langle\cdot\rangle_{z}$ the depth average, a scalar quantity that represents net upwelling or downwelling over the entire profile. Like assumption 1, this assumption only holds when averaged over an ensemble and the water column. In deep convection, there may be convergence near the surface around convecting plumes and horizontal divergence at the mixed layer base. This may result in the glider oversampling downwelling near the surface and undersampling downwelling at depth. However, the rates of convergence and divergence expected for the speeds of convection observed suggest that the glider speed of $20 \mathrm{~cm} \mathrm{~s}^{-1}$ is sufficient to make this bias negligible. To conclude, we do not expect large-scale horizontal convergences (divergences) over the 1000-m profile, which would be required to cause vertical divergences (convergences).

Assumption 3 requires that $w_{w}$ not be statistically different when comparing velocities measured, for example, during glider dives with those during glider climbs. We can require $\left\langle w_{d}(z)+w_{c}(z)\right\rangle$ to be small, where $w_{d}(z)$ is a mean profile of $w_{w}$ averaged over glider dives and $w_{c}$ over climbs.

Based on these assumptions, we considered the following cost functions for the minimization procedure: 


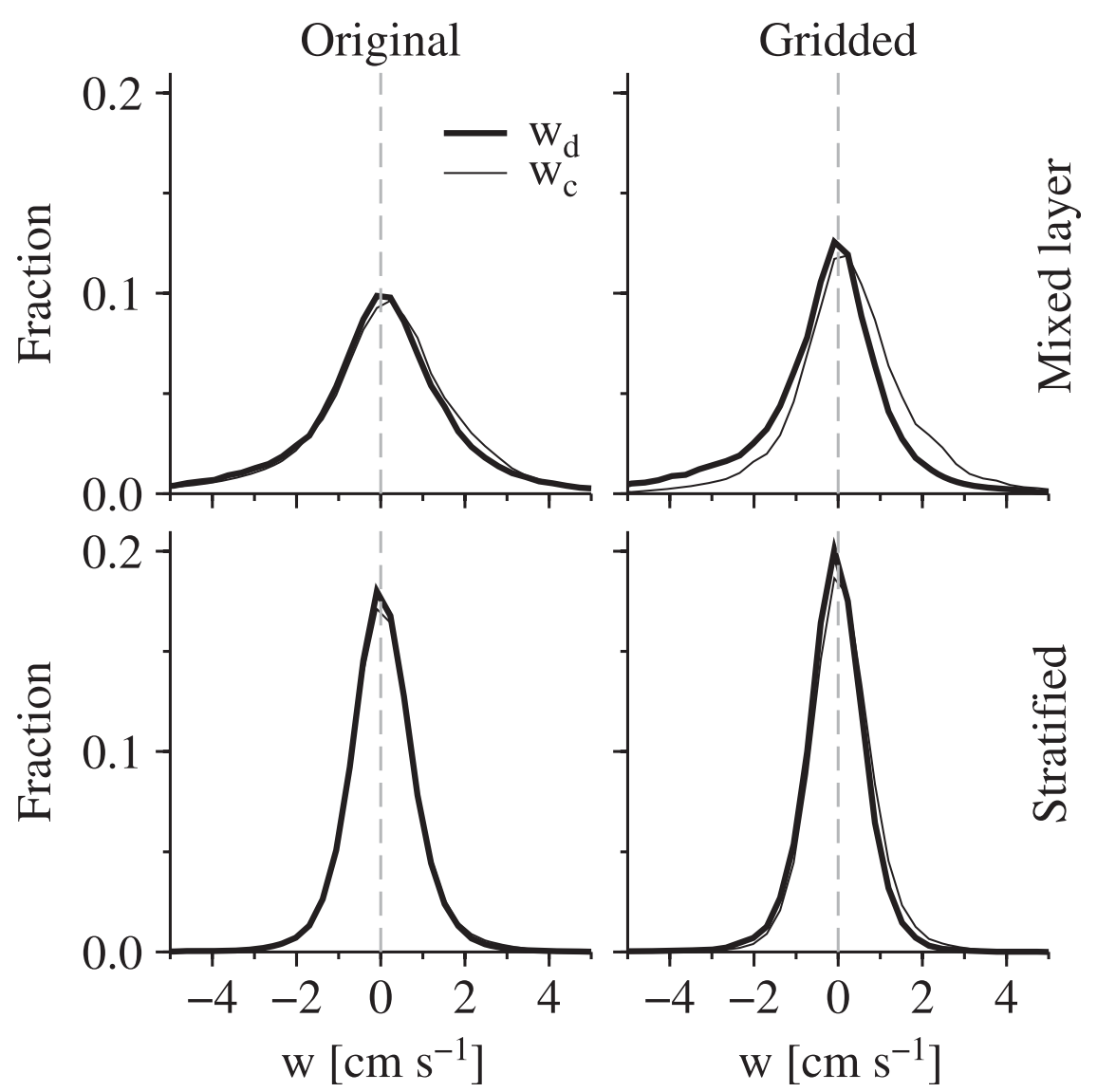

FIG. 7. Resampling glider data onto an evenly spaced depth grid before determining flight parameters results in a downward bias of $w_{d}$ (vertical water velocity from dives) and an upward bias in $w_{c}$. Each of the four subplots shows the histogram of vertical water velocity estimates, separated by whether the estimate was made during a glider climb or dive. Estimates of vertical water velocity from the mixed layer (top left) using the original sampling rate and (top right) using a depth-gridded product. (bottom left),(bottom right) As above, but from the stratified water column. Note that mixed layer depth was calculated as the shallowest depth at which density differs from surface density by at least $0.01 \mathrm{~kg} \mathrm{~m}^{-3}$, when considered in $20-\mathrm{m}$ bins.

(i) vertical water velocity variance

$$
\sum_{t} w_{w}(t)^{2}
$$

(ii) temporal mean profiles from dive and climb

$$
\left\langle\left|w_{d}(z)\right|+\left|w_{c}(z)\right|\right\rangle_{z}
$$

(iii) temporal mean offsets between profiles from dive and climb

$$
\left\langle\left|w_{d}(z)-w_{c}(z)\right|\right\rangle_{z}
$$

(iv) vertical divergence

$$
\left\langle\left|w_{d}(z)-\left\langle w_{d}(z)\right\rangle_{z}\right|+\left|w_{c}(z)-\left\langle w_{c}(z)\right\rangle_{z}\right|\right\rangle_{z}, \quad \text { and }
$$

(v) offsets in dive-climb magnitude or variance,

$$
\left|\left\langle\left|w_{d}(z)\right|-\left|w_{c}(z)\right|\right\rangle_{z}\right|,
$$

where $|\cdot|$ is the magnitude. Mean profiles are calculated over ensembles that are chosen when evaluating the minimization.

In calculating mean profiles, it is important to be aware that sampling statistics of observations from a semiLagrangian instrument such as a glider are affected by the flow regime in which they are made. Trajectories are speeded through downwelling on a dive and through upwelling on a climb. To calculate mean profiles, data are binned in depth so that the relative contributions in each bin are weighted by the glider transit time. The effect of unweighted grid interpolation of glider estimates of vertical velocity is shown in Fig. 7. Vertical velocity 

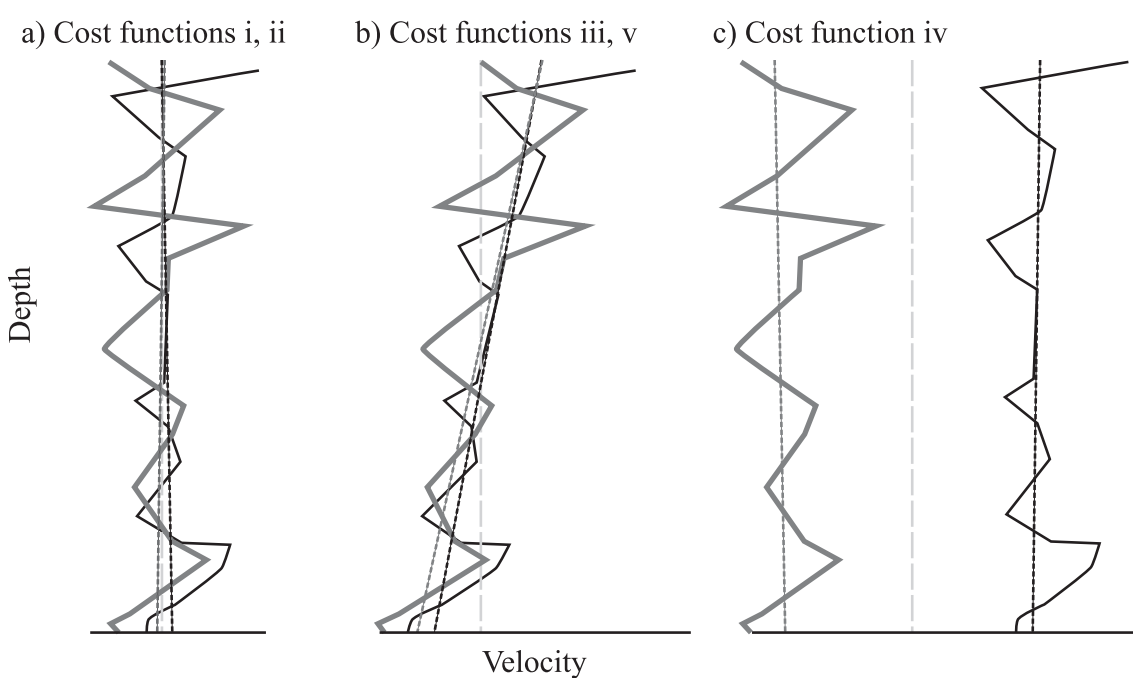

FIG. 8. Choice of minimization procedures, tested for cycles 241-340 from sg014. Each panel shows a schematic of the errors that may remain when applying a particular cost function, corresponding to the list in section $4 \mathrm{a}$. Vertical velocity from dives (black) and climbs (gray) are shown, and straight trend lines have been fit.

estimates were divided by whether the glider was diving or climbing (thick or thin curves) and whether the glider was in the mixed layer or stratified region below (top versus bottom row). On the left are histograms for all sg014 $w_{w}$ estimates, with no gridding applied. On the right are histograms for $\mathrm{sg} 014 w_{w}$ which was interpolated onto a 4-m evenly spaced depth grid. In the mixed layer, gridding accentuates downward motions during dives and upward motions during climbs, resulting in an apparent dive-climb offset. All average profiles calculated for the cost functions were computed by binning measurement time series into depth intervals.

To evaluate the effectiveness of each cost function $w_{w}$, they were minimized over a small ensemble of 60 dive cycles from 23 November to 16 December 2004, during which time the glider traveled roughly $360 \mathrm{~km}$ over ground. All of the parameters were allowed to vary (lift $a$, drag $b$, compressibility $\gamma_{g}$, and volume $V_{0}$ ). It was found that the first two cost functions resulted in a near-zero mean profile of vertical velocity from dives and from climbs, with little vertical divergence (Fig. 8a). They also determined similar values of parameters. The third cost function resulted in a mean dive profile similar to the climb profile, but both had divergence, with net downward transport in the upper $500 \mathrm{~m}$ and net upward transport in the lower $500 \mathrm{~m}$ (Fig. 8b). This satisfies the zero net vertical transport requirement but violates the nondivergence assumption.

The fourth cost function resulted in mean profiles from dive and climb that had little divergence, but the climb profile was uniformly downward at $2 \mathrm{~cm} \mathrm{~s}^{-1}$ and the dive profile was upward at $2 \mathrm{~cm} \mathrm{~s}^{-1}$. This too satisfies the zero net vertical transport, but violates the third assumption that the vertical velocity of the water does not depend on the state of the glider (Fig. 8c). The final cost function behaved similarly to the third in requiring that the difference between the mean profiles of variance from dives be similar those from climbs, but it did not require that the mean profiles of variance be small (Fig. 8b). Thus, we conclude that either $\sum_{t} w_{w}(t)^{2}$ or $\left\langle\left|w_{d}\right|+\left|w_{c}\right|\right\rangle_{z}$ is the best cost function. Because $\left\langle\left|w_{d}\right|+\left|w_{c}\right|\right\rangle$ directly restricts our third assumption, we have used it in subsequent calculations.

\section{b. Optimization procedure}

Before applying the optimization to an entire glider mission, we discard some parts of the glider data as previously described. Recall that $a$ and $b$ can only be distinguished over dive cycles where attack angle $\alpha$ varies. For the Seaglider missions used here, glider pitch $\varphi$, and thus $\alpha$, was varied in the first 50 dive cycles. The minimization procedure is to regress for $a$ and $b$ jointly over the first 50 cycles, then for $\gamma_{g}$ and $\delta V_{0}$ on the full mission (1-663 for $\operatorname{sg} 014$ and 1-617 for $\operatorname{sg} 015)$. These two steps are alternated and iterated until parameter values converge.

After this first series of minimizations, the resulting offset between the dive and climb profiles over the entire mission was still $\sim 0.1 \mathrm{~cm} \mathrm{~s}^{-1}$. Because only changes to lift or drag coefficients can reduce an offset between dive and climb profiles, we fixed $a$ at the value determined, then regressed for $b$ only on the full mission, and then $\gamma_{g}$ and $\delta V_{0}$, iterating these two steps again until the parameters converged. The final value of $b$ was 0.0088 instead of 0.0092 , a change of less than $5 \%$, and the dive-climb offset 


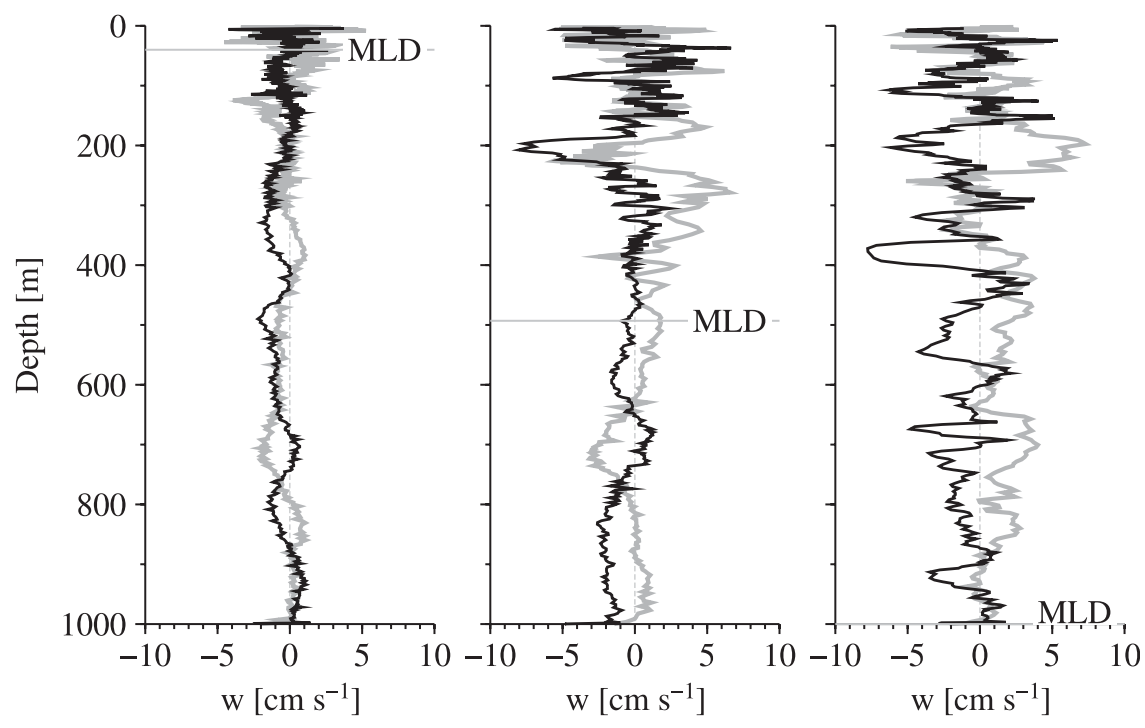

FIG. 9. Sample dive cycle profiles of vertical velocity from a (left) stratified region, (middle) deeply convecting region, and (right) mixed, where the mixed layer depth is around $500 \mathrm{~m}$. Dive profiles (black) and climb profiles (gray) are shown.

was reduced to less than $0.05 \mathrm{~cm} \mathrm{~s}^{-1}$. The parameter set that minimized $\left\langle\left|w_{d}\right|+\left|w_{c}\right|\right\rangle_{z}$ from $\mathrm{sg} 014$ was

$$
\begin{aligned}
a & =0.004 \mathrm{rad}^{-1}, \\
b & =0.0088 \mathrm{~m}^{1 / 4} \mathrm{~kg}^{1 / 4} \mathrm{~s}^{-1 / 2}, \\
c & =2.1 \times 10^{-6} \mathrm{rad}^{-2}, \\
\gamma_{g} & =4.11 \times 10^{-6} \mathrm{dbar}^{-1}, \\
\delta V_{0} & =-12.4 \mathrm{cc},
\end{aligned}
$$

where the new $V_{0}=V_{0, \text { initial }}-\delta V_{0}$. Mean profiles are shown in Fig. 3 (solid lines).

\section{Results: Vertical velocities}

To confirm the validity of the vertical velocity measurement and to estimate its accuracy, we analyze it in the context of theoretical expectations of $w_{w}$ observed in the stratified and unstratified ocean, where dynamics and forcings differ. The velocity observations will be explored further in another paper.

\section{a. Observations}

The two Seagliders in the Labrador Sea observed a wide range of water masses-fresh, cold Arctic waters and warm, salty North Atlantic waters-as well as a range of dynamic regimes. From January to February, mixed layers deepened until there was a less than $0.01 \mathrm{~kg} \mathrm{~m}^{-3}$ range in density over the $1000-\mathrm{m}$ profile. A wide range of processes were observed-from internal waves to buoyancy-forced deeply convecting mixed layers - making the dataset a good choice for evaluating vertical velocity.

Sample dive cycles of vertical velocity show these distinct regimes (Fig. 9). In the stratified profile (Fig. 9a), note the regular, lower-amplitude variations, which are indicative of internal waves. The profile from the 1000-m mixed layer (Fig. 9c) has irregular, larger amplitude spikes in vertical velocity, while profiles with a mixed layer depth around $500 \mathrm{~m}$ highlights the transition between the mixed layer above and the stratified region below (Fig. 9c). Overall, vertical speeds estimated from the Seaglider were $w_{\mathrm{rms}}=0.9 \mathrm{~cm} \mathrm{~s}^{-1}$ in the stratified regions and $w_{\mathrm{rms}}=2.1 \mathrm{~cm} \mathrm{~s}^{-1}$ in the mixed layer.

\section{b. Consistency check}

To evaluate the consistency of vertical water velocity, we first examine the structure of offsets between dive and climb profiles of velocity, then compare observations to theoretical expectations of magnitude in relation to stratification or forcing, and finally examine the spectral structure of velocity.

The structure of the dive-climb offset for the entire mission appears random (Fig. 10a). Offsets were calculated as the difference between mean profiles of vertical velocity from dives and climbs, where the mean profiles were averaged over ensembles of 20 dive cycles. Offsets were distributed around zero (mean \pm standard deviation $=0.01 \mathrm{~cm} \mathrm{~s}^{-1} \pm 0.5 \mathrm{~cm} \mathrm{~s}^{-1}$ ), with no clear bias relating either to depth in the water column or mixed layer depth. While magnitudes of the offset are higher in 

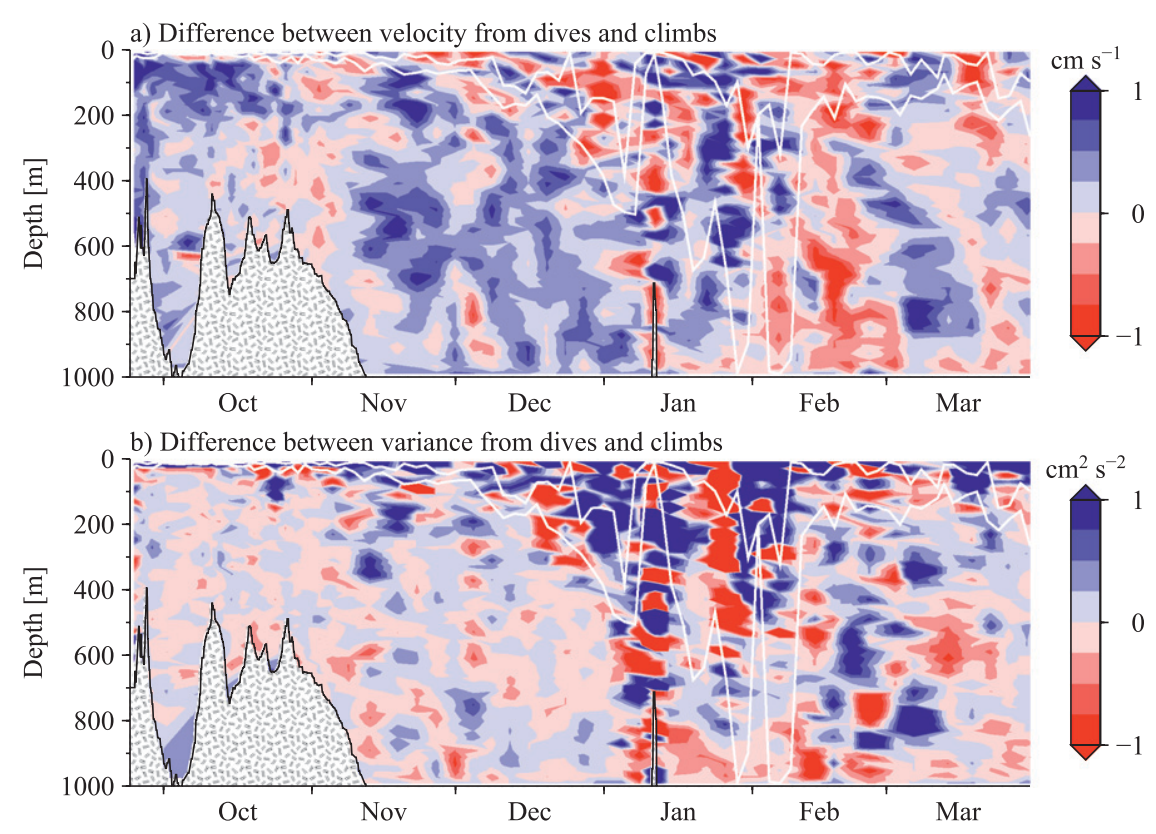

FIG. 10. Structure in the offset between (a) vertical velocity estimates and (b) vertical velocity variance from glider dives and climbs. Here, the offset is determined between ensembles of 20 profiles in 20-m-depth bins, as the mean from dives minus climbs. Regions where the average velocity profile from dives is more upward than from the climb (red) are shown. Bathymetry is stippled. Minimum and maximum mixed layer depths observed during each ensemble are overlaid (white).

the deep mixing regions, velocities there are also higher. Similarly, the dive-climb offset in vertical velocity variance was calculated, again showing little structure and higher values in the mixed layer, as before (Fig. 10b).

In the stratified water column, internal wave energy is expected to dominate. While the Seaglider's slanted profiles and slow speeds are unable to resolve the frequency and wavenumber of wave motions, the magnitude of the internal wave energy can be estimated, and it is expected to scale with stratification as $1 / N$, where $N$ is the buoyancy frequency. For the typical stratified ocean, the relationship is determined by integrating the GarrettMunk spectrum

$$
\left\langle w^{2}\right\rangle=0.25 N_{0} / N
$$

where $N_{0}=5.3 \times 10^{-3} \mathrm{rad} \mathrm{s}^{-1}$ is a reference buoyancy frequency, and the constant factor 0.25 results from constants in the GM76 spectrum (Munk 1981). The theoretical scaling and glider-estimated vertical velocity agree for lower $N$ (Fig. 11). Above $N \approx 1.5 \times 10^{-3} \mathrm{rad} \mathrm{s}^{-1}$, in the thermocline, the scaling breaks down. However, the thermocline is not the canonical ocean-it is near-air-sea forcing and also a waveguide. The waveguide results from a peak in $N$ below the mixed layer; this peak can trap energy with a higher frequency (Desaubies 1973; Munk 1980). Energy levels are elevated in the thermocline below the mixed layer and do not conform to the expectations given in (11). Below the thermocline in the stratified water column $N<1.5 \times 10^{-3} \mathrm{rad} \mathrm{s}^{-1}$, vertical speed scales with the inverse of stratification $\left\langle w^{2}\right\rangle \sim 1 / N$. The same calculation was done for untuned glider data (Fig. 11, $\times$ symbols). Note the higher $\left\langle w^{2}\right\rangle$ at low $N$ $\left(<0.001 \mathrm{rad} \mathrm{s}^{-1}\right)$, resulting from the nonzero mean $w$ in the untuned glider data.

In the unstratified mixed layer, primary energy sources are winds and buoyancy flux from the atmosphere. We expect that vertical speeds scale with heat flux or wind. Comparing the time series of rms vertical velocity averaged within the mixed layer and over 1-day periods to these two surface forcings along glider trajectories, we found that velocities lag the forcing at 0.6 and 0.7 days, and have $r=0.65$ and $r=0.58$ correlation coefficients, respectively (see Fig. 12). This positive relation between vertical speeds and forcing indicates that strong surface forcing generates kinetic energy in the surface mixed layer, resulting in higher water speeds. The lags are similar to those found by Steffen and D'Asaro (2002), who compared vertical velocities measured by Lagrangian floats in the Labrador Sea convection. The same calculation was made for untuned glider data. While the lags between atmospheric forcing and ocean response were the same, the correlation coefficient was reduced (Fig. 12, gray). 


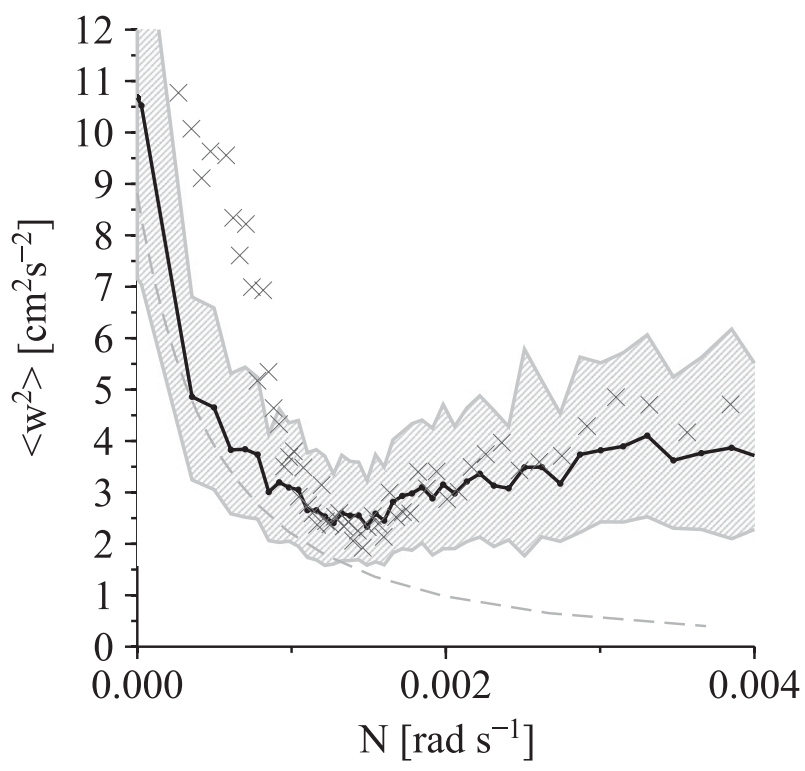

FIG. 11. Dependence of vertical speed squared on stratification, given by buoyancy frequency $N$. Glider estimates of $w_{w}$ were binned by the collocated estimate of $N$. Each point represents approximately 3000 measurements of $w_{w}$. The shaded interval is the $95 \%$ confidence interval. The $\times \mathrm{s}$ are similarly calculated averages of $w_{w}^{2}$ but using the original, untuned flight parameters. The dashed gray curve is $\left\langle w^{2}\right\rangle \approx 0.25 N_{0} / N$ for $N_{0}=5.3 \times 10^{-3} \mathrm{rad} \mathrm{s}^{-1}$.

Frequency spectra were calculated from profiles of vertical velocity in and below the mixed layer. In the internal wave band, frequencies between $f$ the Coriolis frequency and $N$ the GM model predict a white (flat) spectrum for vertical velocity. Above $N$, turbulence dominates and the spectrum is described by the Kolmogorov spectrum with a $-5 / 3$ slope. To calculate spectra, data were first subsampled to a constant sampling interval $(20 \mathrm{~s})$ and then separated into continuous profiles of at least 256 data records. Each record is then $85 \mathrm{~min}$ long or about $350 \mathrm{~m}$ in vertical extent. There were 34 such profiles in the mixed layer and 53 in stratified water. In the mixed layer, then, this represents data from mixed layers at least $350 \mathrm{~m}$ deep. Periodograms were averaged to produce the spectra.

Energy in the mixed layer is a decade higher than below the mixed layer, and slopes are red everywhere (Fig. 13). The average buoyancy frequency in the mixed layer $N=6 \times 10^{-4} \mathrm{rad} \mathrm{s}^{-1}$, while in the stratified profiles it is $N=0.0015 \mathrm{rad} \mathrm{s}^{-1}$. The overall range of frequencies for which the spectrum is calculated is much lower than that in Merckelbach et al. (2010). This is because our sample rate was variable and lower overall than that for the Slocum glider missions they used. We restricted the calculation to data sampled at least every $20 \mathrm{~s}$, which is only a few hours long, and thus cannot resolve the lowest frequencies. Using a lower threshold sample rate (40 s)

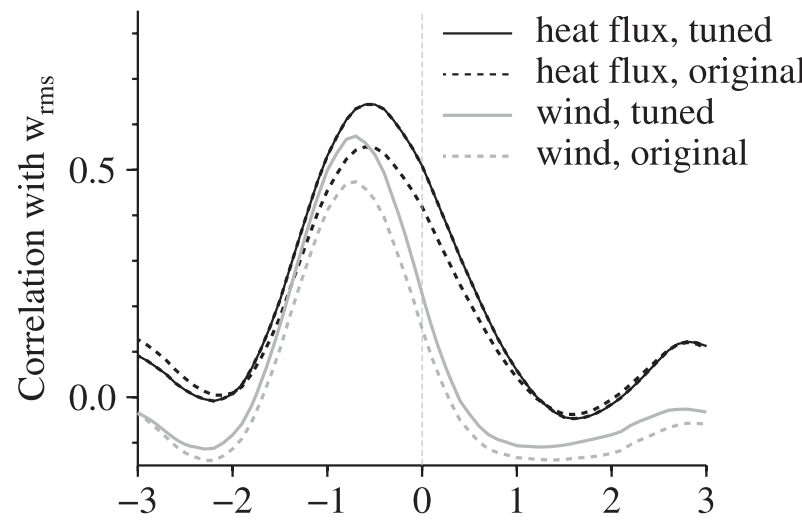

Time shift [days], ocean leading atmosphere

FIG. 12. Lag correlation plots between $w_{\text {rms }}$ and surface heat flux and wind speed. Time series of $w_{\text {rms }}$ were averaged daily in the mixed layer and compared with the incident surface forcing. Data using the original parameters (gray) and with the tuned parameters (black) are shown. Correlations improved with tuning, though the lag remained the same.

allowed more data to be used, but did not resolve the high-frequency rolloff necessary to estimate instrument noise (next section). The slopes observed here are different than those in Merckelbach et al. (2010), who found -3 slopes until $10^{-2} \mathrm{rad} \mathrm{s}^{-1}$ (their highest average buoyancy frequency), and a $-5 / 3$ or -1.66 slope at higher frequencies until the high-frequency rolloff at $10^{-1.4}$ $\operatorname{rad~s}^{-1}$. Here we find slopes closer to -1.4 for the mixed layer data between $10^{-2.5}(0.0032)$ and $10^{-1.1}(0.08)$ $\mathrm{rad} \mathrm{s}^{-1}$. For the stratified data, the lower-frequency slope [below $10^{-1.3}(0.05) \mathrm{rad} \mathrm{s}^{-1}$ ] is around -2 and above, about -1 .

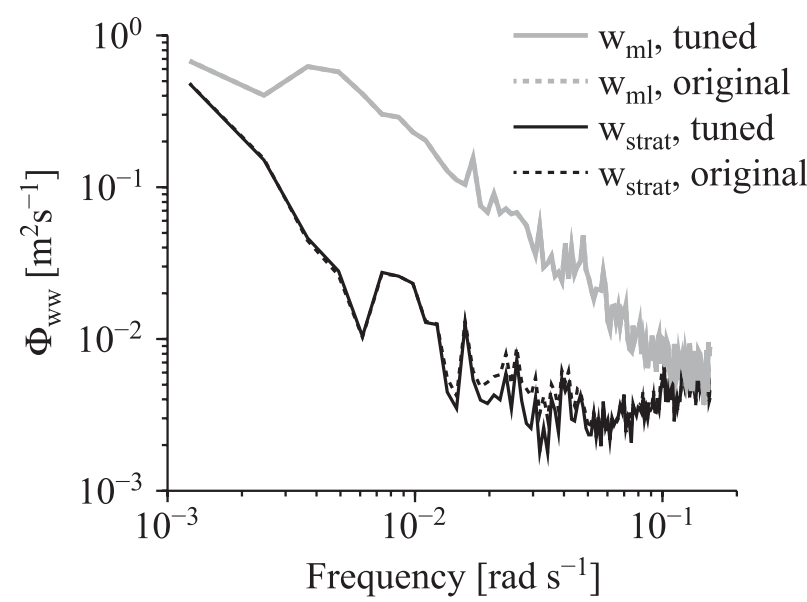

FIG. 13. Frequency spectra of vertical water velocity. Profiles from entirely within the mixed layer (black) and the stratified ocean (gray) are shown. Dashed curves of the same color are spectra calculated using the original, untuned flight parameters. Note that the difference is only apparent in the stratified case. 
In summary, the magnitudes of vertical velocity are higher in the mixed layer than stratified regions, and loosely agree overall with theoretical expectations for the two regimes. Theoretical slopes for spectra are not obtained, but spectra are not unreasonable. Note, however, that spectra from untuned glider data are nearly indistinguishable from the tuned spectra. The only difference is apparent in the stratified water at frequencies from $10^{-2}$ to $10^{-1.5} \mathrm{rad} \mathrm{s}^{-1}$. This is because our correction primarily fixed the mean and dive-climb offset, which are lower frequencies than the spectra resolve.

\section{c. Error estimates}

Errors in $w_{w}$ are due to instrument noise affecting $w_{\text {meas }}$ and imperfections in the glider flight model predicting $w_{\text {stdy }}$. Errors can be estimated by examination of 1) high-frequency noise levels, 2) mean values (following Merckelbach et al. 2010), 3) the offset in vertical velocities between mean dive and climb profiles, and 4) the offset in vertical velocity variance between mean dive and climb profiles.

The high-frequency noise level is calculated by integrating the frequency spectrum of vertical velocity above the high-frequency rolloff. For profiles in the stratified water, this rolloff occurs above $N>0.04 \mathrm{rad} \mathrm{s}^{-1}$, where instrument noise increases (Fig. 13a). Using this technique, the noise estimate from this method is $\pm 0.5 \mathrm{~cm} \mathrm{~s}^{-1}$.

For comparison with Slocum accuracy in Merckelbach et al. (2010), we use the third method as detailed in their section $4 \mathrm{~d}$. The mean of vertical velocities is $0.3 \mathrm{~mm} \mathrm{~s}^{-1}$ for both sg014 and sg015. Mean values over 3-day periods are $0.1 \pm 2$ and $0.3 \pm 6 \mathrm{~mm} \mathrm{~s}^{-1}$. Over 50 -m depth bins, the mean is -0.04 and $0.05 \mathrm{~mm} \mathrm{~s}^{-1}$. Using these values, the systematic error is approximately $\pm 2 \mathrm{~mm} \mathrm{~s}^{-1}$. Over the same 3-day periods, we compare the vertical velocity from dives with climbs. The offset of $w_{d}-w_{c}$ is $-3 \pm 2$ and $2 \pm 5 \mathrm{~mm} \mathrm{~s}^{-1}$ for $\mathrm{sg} 014$ and $\mathrm{sg} 015$, respectively. Over the same $50-\mathrm{m}$ depth bins, the offset is $0.007 \pm 0.9$ and $-2.3 \pm 0.9 \mathrm{~mm} \mathrm{~s}^{-1}$ for noise levels in the spectrum.

For the entire mission, the mean vertical velocity from climbs is $-0.048 \mathrm{~mm} \mathrm{~s}^{-1}$ and from dives is $0.53 \mathrm{~mm} \mathrm{~s}^{-1}$. Variance is nearly identical from climbs $\left(1.6 \mathrm{~cm} \mathrm{~s}^{-1}\right)$ and from dives $\left(1.59 \mathrm{~cm} \mathrm{~s}^{-1}\right)$. We conclude that Seaglider vertical velocity accuracy is $0.5 \mathrm{~cm} \mathrm{~s}^{-1}$.

\section{Summary}

We detailed a methodology to calculate vertical water velocities from Seaglider measurements of salinity, temperature, and pressure based on a steady flight model. In evaluating the procedure, we described how flight parameters affect estimates of vertical velocity: induced drag and volumetric thermal expansion have little effect; lift and drag change the offset between profiles estimated from dives and climbs. Lift and drag coefficients were found to have compensating effects, which could only be separated over profiles with a range of pitches. In solving the minimization problem for flight parameters, five choices of the cost function were tested. Three of the cost functions resulted in unrealistic profiles of vertical velocity; there was little difference between the other two cost functions, and both produced reasonable estimates of velocity. We chose to use $\left\langle\left|w_{d}\right|+\left|w_{c}\right|\right\rangle_{z}$ because it directly constrains the actions of the flight parameters. In applying the cost function, it was first minimized first over the 50 dives where pitch varied, in order to fix the lift coefficient, and then over the entire mission. The resulting offset between the mean dive and climb profiles was very small $\left(<0.05 \mathrm{~cm} \mathrm{~s}^{-1}\right)$, and the pattern of offsets over the course of the mission and was reassuringly random in depth.

Vertical velocities were compared with the theoretical Wentzel-Kramers-Brillouin (WKB) scaling from Munk (1981) and found agreement in the weakly stratified deep ocean, though velocities were elevated above expectations in the thermocline. Vertical speeds in the mixed layer were positively related to the surface forcings, winds and buoyancy flux. Using the high-frequency rolloff in the vertical velocity spectrum gives an estimate of the noise, approximately $\pm 0.5 \mathrm{~cm} \mathrm{~s}^{-1}$. However, comparing with the Slocum gliders in Merckelbach et al. (2010), and using the same estimate of accuracy, the Seaglider accuracy is $2 \mathrm{~mm} \mathrm{~s}^{-1}$ compared to Slocum's $4 \mathrm{~mm} \mathrm{~s}^{-1}$.

Comparing our methodology with that in Merckelbach et al. (2010), the flight models differ slightly. In the case of Seaglider, the parameterization has been determined from wind test experiments to include a dependence on $q$, which is multiplied by our drag coefficient. In addition, we use a single lift coefficient $a$ to represent total lift by the body and wings of the instrument. Comparing the flight parameters, our drag coefficient is $b q^{-1 / 4}$ multiplied by $l^{2}$, giving $0.0088(1024 / 2) \times\left(0.2^{2}\right)(3.24) \approx 0.58 \mathrm{~m}^{2}$, where they have $\left(C_{D_{0}}+C_{D_{1}} \alpha^{2}\right) S=\left(0.1+2.88 \alpha^{2}\right)(0.1) \approx 0.0186 \mathrm{~m}^{2}$ for $\alpha=3^{\delta}$. These are of similar size. Our lift coefficient of $a=0.004$ is multiplied by an $l^{2}=3.24 \mathrm{~m}^{2}$ giving $\approx 0.013 \mathrm{~m}^{2} \mathrm{rad}^{-1}$, which is smaller than their $\left(a_{h}+a_{w}\right) S=$ $(2.4+3.7)(0.1)=0.61$. Differences in glide angle $\left(26^{\circ}\right.$ for Slocum versus $16^{\circ}$ for Seaglider) and flight speed (32 versus $23 \mathrm{~cm} \mathrm{~s}^{-1}$ ) do not account for this difference. However, Merckelbach et al. (2010) uses lift coefficients determined from another source, and then increases the lift coefficient in order to reduce errors in horizontal velocity estimates. 
One major difference in the methodology between Merckelbach et al. (2010) and this paper is that here, rather than fixing our lift coefficient from other results, we determine the lift coefficient using the optimization procedure. The primary reason we were able to do this is that the Seagliders were piloted through a range of pitches near the beginning of the mission, allowing us to separate the effects of lift and drag on vertical velocity. The Slocum gliders used in Merckelbach et al. (2010) attempted a $26^{\circ}$ slope for the whole mission. To a large extent, changes in lift and drag coefficients can compensate each other, however small the introduced errors would be as glide angle varies. In addition, high-frequency noise levels are best determined with a relatively high sample rate $(<0.1 \mathrm{~Hz}$ or every $10 \mathrm{~s})$. Our method also allows a thermal expansion effect, which was negligible for the Labrador Sea gliders used here. However, the vertical temperature stratification was quite weak, and it is possible that in a more stratified region, the thermal expansion could have an effect.

Vertical velocities have been historically tricky to measure because of their low amplitudes. Seagliders can do it within $0.5 \mathrm{~cm} \mathrm{~s}^{-1}$, returning full 1000-m profiles of vertical velocity. Though the Seaglider is unable to resolve tides in the typical fashion (using frequency or wavenumber spectra), this technique is appropriate for application to mixed layers and velocity in mesoscale structures-fronts and eddies-where vertical velocities may be both large and important. The accuracy and resolution may be improved by incorporating the effect of rolls into the flight model, or by using an unsteady flight model allowing acceleration. While outfitting Seaglider with an instrument that can independently measure vertical water velocity could help verify glider measurements of $w_{w}$, such an instrument may be large or impact the hydrodynamic shape of the Seaglider, two things that could render the flight model inappropriate.

While the estimate of velocity accuracy from Seaglider is quite good, it is worth mentioning that interpretation of these data may not be straightforward. The Seaglider slant profile and translational speed influence the measurements. In the stratified ocean, for all but the highest frequency waves and plumes, the glider is essentially making vertical profiles. The $f / N$ scaling for the aspect ratio of motion is typically small compared with the glider 1:3 path. In the lower stratified mixed layers, this may not be the case. The glider may observe 200-m-wide plumes, which descend to $1000 \mathrm{~m}$. For these, the glider may be seeing horizontal structure. A more complete application of glider vertical velocity data is in progress.

Acknowledgments. The authors thank Eric D'Asaro for enlightening discussions of the Labrador Sea and vertical velocities, as well as Eric Kunze, Nicholas Beaird, and Amanda Gray for helpful insights. RRH was supported ONR Grant N00014-08-1-0446. The Seaglider project was supported by NSF Grant OCE-0550584. EFW was supported by a National Science Foundation Graduate Research Fellowship for a part of this work.

\section{APPENDIX}

\section{Effect of a Pitch Reading on $w$ (sg015)}

Glider sg015 was found to have a faulty pitch reading, likely resulting from electrical interference between the pitch sensor and other sensors on board. For Seaglider, this was diagnosed by estimating the relationship between measured pitch and pitch control. The glider estimates a desired pitch angle based on its distance from the target latitude and longitude and the flight model. Variations in pitch affect the speed over ground that the glider makes, because shallower pitch angles result in more distance covered over ground. For a target that is quite close, a steeper pitch angle would be required. To meet that angle, it adjusts pitch control, which refers to the distance fore and aft that the battery pack moves within the glider body to set the pitch. The gain is approximately $12^{\circ}-15^{\circ}(1 \mathrm{~cm})^{-1}$ movement. Because pitch also depends on buoyancy and the marginal volume (VBD), we calculate the linear relationship between the observed pitch and the predicted control elements for $\operatorname{sg} 014$ to check the stability of the relationship

$$
\theta=\mathbf{A}\left[\begin{array}{c}
\mathbf{I} \\
\theta_{\text {control }} \\
B \\
v_{c}
\end{array}\right],
$$

where $\mathbf{A}$ are the coefficients $\left(\mathbf{A}_{1}\right.$ being a mean contribution), $\mathbf{I}$ is an identify vector, $\theta_{\text {control }}$ is the pitch control, $v_{c}$ is the volume change produced by the VBD, and $B$ is the glider's buoyancy.

Pitch control $v_{c}$ ranges between $\pm 2 \mathrm{~mm}$, causing a pitch angle of $\pm 40^{\circ}$ in sg014. Buoyancy ranges between $\pm 200 \mathrm{cc}$. VBD ranges from -100 to +300 . There is a positive relationship between buoyancy and VBD. The vector $\mathbf{A}$ calculates the dependence of measured pitch on each of these values. For a properly working sensor, we expect the relationship, defined by $\mathbf{A}$, to remain approximately constant over the course of the mission.

Estimates of $\mathbf{A}$, calculated over ensembles of 20 dive cycles, were fairly constant in the mean, though some dependence on pitch control is seen (Fig. A1). In the latter half of the mission, when the glider was experiencing deep 

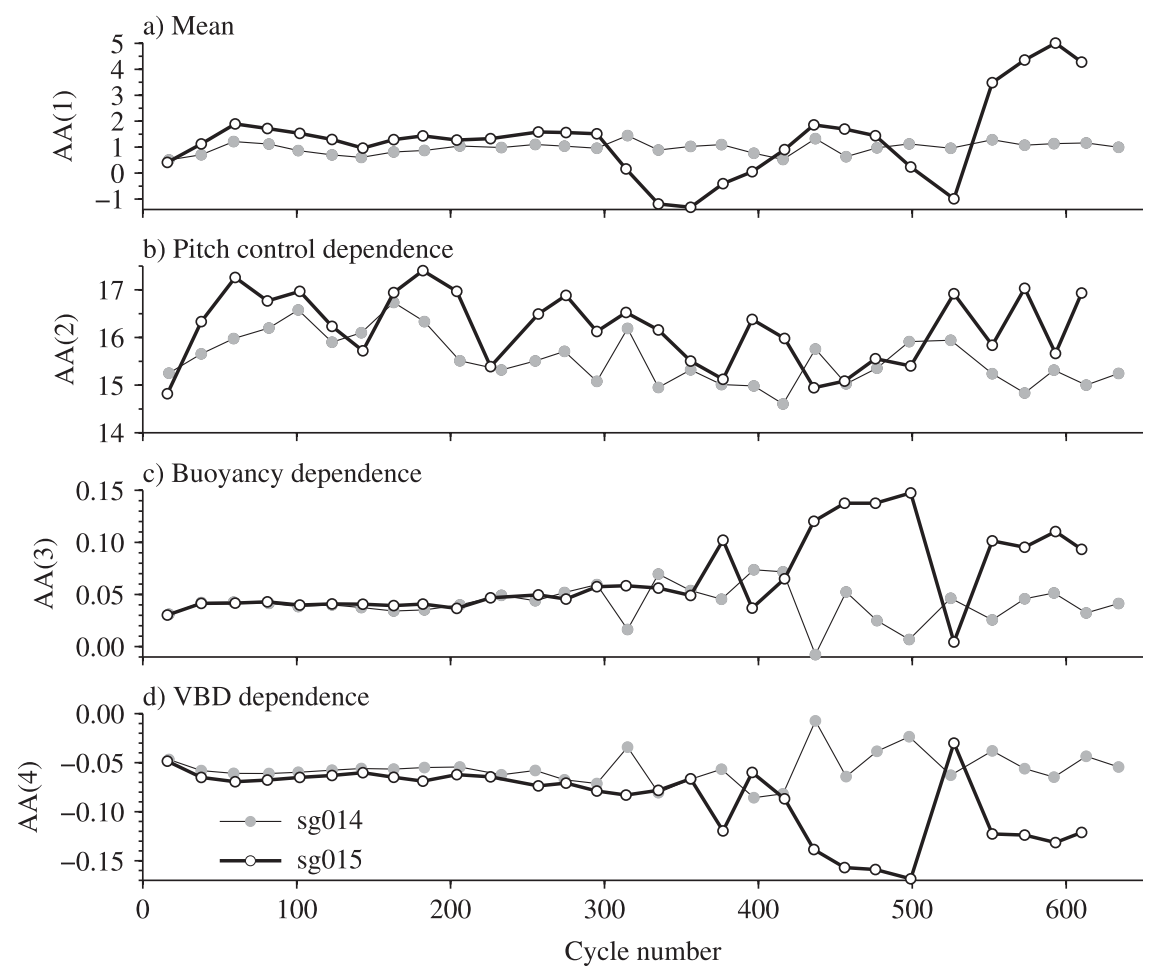

FIG. A1. Stability of measured pitch related to (a) mean, (b) pitch control, (c) buoyancy, and (d) VBD, as described by Eq. (A1), for sg014 (gray) and sg015 (black). The mean is relatively steady for $\mathrm{sg} 014$ and for $\mathrm{sg} 015$ before dive cycle 300 . After dive cycle 300 , the mean for sg015 ranges from -2 to 5 .

convection (cycles 350-550), buoyancy and VBD dependence appear more variable but are in fact compensating. The variability in $\mathbf{A}(1)$ in the latter half of sg015's mission indicates a changing relationship between pitch control and measured glider pitch. Unlike the buoyancy problems encountered for Slocum gliders in Merckelbach et al. (2010), this error began partway through the mission (rather than being continuously

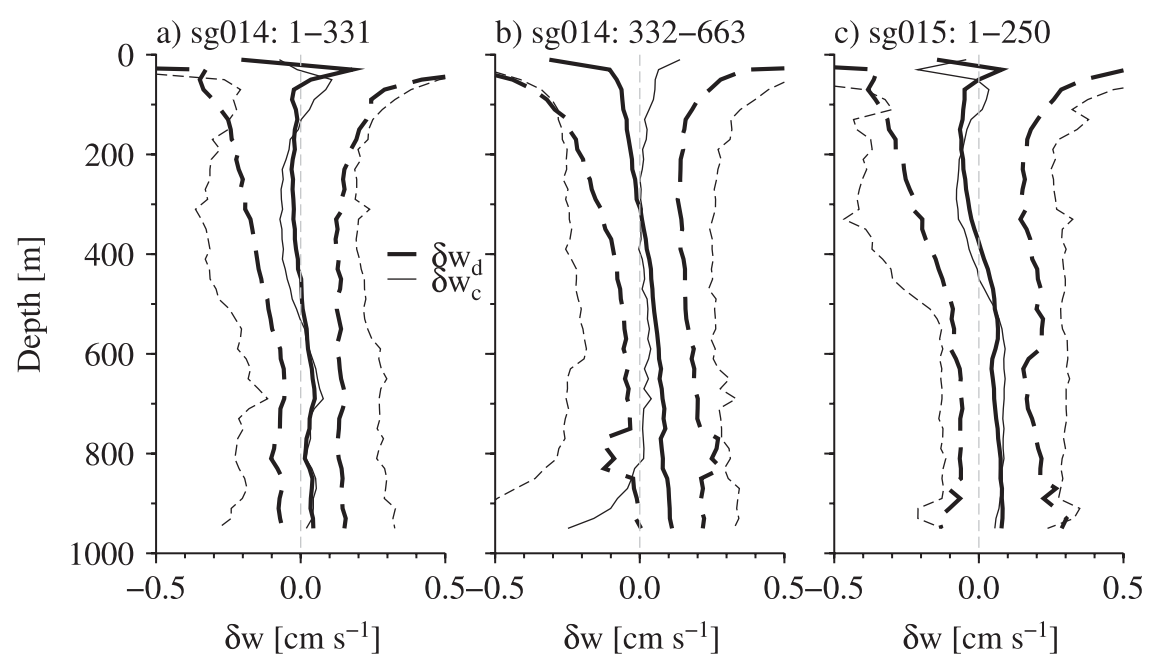

FIG. A2. Mean change in profiles of vertical velocity $\left(w_{w}\right)$ resulting from substituting modeled pitch, calculated from Eq. (A1) for measured pitch. (a) sg014 cycles 1-331, (b) sg014 cycles 332-663, and (c) sg015 cycles 1-308. Effect of pitch correction procedure on mean profiles of vertical velocity from dives (black) and climbs (gray) and with standard deviations in dashed lines of the same color. The overall change is $<0.1 \mathrm{~cm} \mathrm{~s}^{-1}$. 
present), and errors in the pitch readings sometimes coincided with electrical activity by other sensors.

To improve the estimate of vertical velocity from sg015, we replace measured pitch with a calculated pitch using A. In doing so, we are assuming that the pitch control is accurately adjusting the glider pitch and that the relationship between glider pitch and control is constant for the duration of the mission.

To evaluate the appropriateness of corrected pitch as an input for the glider flight model, we apply it to profiles where the pitch readings appeared accurate. Those profiles included all 1-663 of sg014 (divided into two segments of 1-331 and 332-663) and the first half of sg015's mission (1-308). Vertical velocities were calculated using the corrected pitch and measured pitch, and then differenced. Differences are largest near the surface where pitch changes rapidly (Fig. A2). There also appears to be a mean offset in vertical velocity from corrected pitch between the dive and climb measurements. The offset is on order of $0.1 \mathrm{~cm} \mathrm{~s}^{-1}$, with climbs having greater mean upward velocity.

Correcting sg015's pitch in this way for the latter half of the record will likely result in a mean offset between the dive and climb profiles. Vertical velocity error is increased by about $0.1 \mathrm{~cm} \mathrm{~s}^{-1}$.

\section{REFERENCES}

Desaubies, Y. J. F., 1973: Internal waves near the turning point. Geophys. Astrophys. Fluid Dyn., 5, 143-154.

Eriksen, C. C., T. J. Osse, R. D. Light, T. Wen, T. W. Lehman, P. L. Sabin, J. W. Ballard, and A. M. Chiodi, 2001: Seaglider: A long-range autonomous underwater vehicle for oceanographic research. IEEE J. Oceanic Eng., 26, 424-436.

Frajka-Williams, E., 2009: The spring phytoplankton bloom and vertical velocities in stratified and deep convecting Labrador Sea, as observed by Seagliders. Ph.D. thesis, College of Ocean and Fishery Sciences, University of Washington, 140 pp.

Hubbard, R. M., 1980: Hydrodynamics technology for an Advanced Expendable Mobile Target (AEMT). Applied Physics Laboratory, University of Washington Tech. Rep. APL-UW 8013, 34 pp.

Kanamitsu, M., W. Ebisuzaki, J. Woollen, S.-K. Yang, J. J. Hnilo, M. Fiorino, and G. L. Potter, 2002: NCEP-DOE AMIP-II Reanalysis (R-2). Bull. Amer. Meteor. Soc., 83, 1631-1643.

Merckelbach, L., D. Smeed, and G. Griffiths, 2010: Vertical water velocities from underwater gliders. J. Atmos. Oceanic Technol., 27, 547-563.

Munk, W. H., 1980: Internal wave spectra at the buoyant and inertial frequencies. J. Phys. Oceanogr., 10, 1718-1728.

_ 1981: Internal waves and small-scale processes. Evolution of Physical Oceanography: Scientific Surveys in Honor of Henry Stommel, B. A. Warren and C. Wunsch, Eds., MIT Press, 264-291.

Steffen, E. L., and E. A. D'Asaro, 2002: Deep convection in the Labrador Sea as observed by Lagrangian floats. J. Phys. Oceanogr., 32, 475-492. 